\title{
Daño miocárdico secundario a asfixia neonatal grave
}

\author{
Aldo Bancalari M. ${ }^{3}$; Carlos Otero V. ${ }^{2}$; Paulina Bello R. ${ }^{3}$; \\ Gonzalo Soto G. ${ }^{\text {; }}$ Enzo Pandolfi B. ' ; Luis León A.
}

\section{Myocardial damage following neonatal asphyxia}

\begin{abstract}
Sixteen newborn infants with severe asphyxia were prospectively studied for evidence of secondary myocardial damage and, in that case, their clinical findings. Myocardial damage was diagnosed in three term newborn infants of adequate weight for gestacional age $(18,7 \%)$ by means of serial electrocardiograms taken in the first 72 hours of life. Two of them showed evidence of difusse subendocardial ischemia and a third one showed electrocardiographic signs suggesting necrosis of the left ventricular posteroinferior wall. Neither creatinephosphokinase serum activity nor its muscle brain MB iscenzyme were useful in the identification of myocardial damage. All three affected neonates developed respiratory distress syndrome without signs of cardiac failure and one of them died. The histopathological study of this late one showed localized hemorrhage of the papiltary muscles and interventricular septum. These finding underscore the need of serial electrocardiograph ic recordings in newborns with severe asphyxia. sincecardiac dysfunction may inadvertently occur under the appearence of respiratory distress svindrome.

\{Key words: mvocardium, ischemia, asphyxia neonatorum.
\end{abstract}

La relación entre asfixia neonatal grave $y$ disfunción miocárdica fue descrita en $1961^{1}$ y ampliada posteriormente con tres recién nacidos de término con insuficiencia cardiaca congestiva y falla ventricular izquierda, que no eran portadores de enfermedad cardiaca congénita ${ }^{2}$. La asfixia neonatal grave puede determinar grados variables de disfunción mjocárdica, manifestándose como isquemia transitoria del músculo cardíaco que puede terminar en infarto; insuficiencia tricuspidea; insuficiencia cardiaca congestiva; edema agudo pulmonar y choque cardjogénico ${ }^{1-8}$. Estas manifestaciones, inicialmente, pueden pasar inadvertidas o confundirse con us síndrome de dificultad respiratoria ${ }^{2,3}$. Por este motivo es importante reconocer precozmente el daño miocárdico secundario a asfixia perinatal, a fin de instaurar las medidas que permitan una mejoría más rápida del recién nacido y su pronóstico. El objetivo del presente estudio fue buscar y describir evidencia clínica y de labo-

1. Hospital Guillermo Grant Benavente, Concepción

2. Hospital Guillermo Grant Benavente, Concepción.

3. Becada de Pediatría. Hospital Guillermo Grant Benavente, Concepción.

4. Químico-Farmacéutico. Laboratorio Central. Hospital Guildermo Grant Benavente, Concepción. ratorio de dario miocárdico en recién nacidos con asfixia neonatal grave.

\section{Material y Método}

Se estudiaron en forma prospectiva 16 recién nacidos de pretérmino y término, que a lo largo de un año ingre. saron a la Unidad de Cuidado Intensivo Neonatal del Servicio de Pediatría del Hospital Guillermo Grant Benavente de Concepción con asfixia neonatal grave, definida según puntuación de Apgar en el primer minuto $\leqslant 3$ y $\$ 5$ a los cinco minutos. Durante las primeras 24 horas de vida un cardiólogo pediátrico efectuó a cada uno de ellos examen cardiovascular, repitiéndolo si se consideraba necesario, según evolución, junto con medición de la presión venosa central (PVC), a través de un catéter en la vena umbilical de acuerdo a técnicas establecidas ${ }^{9}$; medición de la presión arterial con Doppler transcutảneo modelo 801-B, electrocardiograma (EOG), durante las primeras 72 horas de vida $y$, luego, según evolución, telerradiografía de tórax; mediciones de actividad enzimática de creatinfosfoquinasa (CPK), isoenzima MB (CPK-MB), deshiơrogenasa láctica (DHL) y transaminasas glutámico axaloacética (TGO) y pirúvica (TGP), todas efectuadas con espectrofotómetro Shimatzu modelo CL 720 a $25^{\circ} \mathrm{C}$ de temperatura. Además se controlaron glicemia, calcemia, gases arteriales, electrolitos plasmáticos, hemograme y uremia. Fn otros dieciséis recién nacidos de término normales, que constituyeron el gupo control, se efectuaran los mismos exámenes, con excepción de la modición de la PVC, por ser un examen iyvasivo, no 
carente de riesgos. El estudio contó $\infty$ n la aceptación de las madres de los recién nacidos involuctados y la aprobación del Comité de Investigación Científica Clínica del Hospital Gujllermo Grant Benavente de Concepción.

\section{Resultados}

De los 16 recién nacidos con asfixia, 11 eran de término y 5 de pretérmino, su peso promedio era $2.680 \mathrm{~g}$, rango entre 1330 y $4200 \mathrm{~g}$ vs. $3300 \mathrm{~g}$ en los controles. Los promedios de la puntuación de Apgar fueton 2,0 y 4,5 al minuto y los cinco minutos de vida respectivamente vs, 9 y 10 en los controles y la presión arterial sistólica 58 vs. $67 \mathrm{mmHg}$. Catorce de los nif̃os asfixiados evolucionaron con dificultad respiratoria, no pesquisándose en ninguno alteraciones cardíacas mediante la auscultación. En un caso se detectó hipoglicemia y en cuatro hipocalcemia. En 8 (50\%) no se observó acidosis metabólica severa, pero habian recibido bicarbonato de sodio por vía endovenosa durante las maniobras de reanimación. Las radiografías de torax mostraban a]teraciones en $10(63 \%)$ de los casos; en forma de infiltrado intersticial bilateral en nueve de ellos, e jmágenes sugerentes de aspiración me. conial en uno. El resultado de las mediciones de CPK, CPK-MB, DHL y transaminasas se expone en la tabla. Tres de los 16 recjén nacidos con asfixia tuvieron alteraciones electrocardiográficas compatibles con dafo miocárdico isquémico, correspondiendo según el orden de aparición a los casos 3,9 y 15 , todos recién nacidos de término adecuados a la edad gestacional y se describen a continuación:

El caso 3 pesó al nacer 3400 g, Apgar 1 y 5 al minuto y cinco minutos de vida respectivamente. Presión arterial sistólica $55 \mathrm{mmHg}$ y presión verosa centrál $6 \mathrm{~cm}$ de $\mathrm{H}_{2} \mathrm{O}$. La telerradiografía de tórax reveló infiltrado intersticial pulmonar difuso, sin cardiomegalia. CPK total 527 UI/1 y fracción MB 33,5 UI/I (6,3\%). En el electrocardiograma se inscribió onda $q$ anormal en $\mathrm{D}_{2}-\mathrm{D}_{3} \mathrm{y}$ avF, con elevación del segmento ST en $\mathrm{D}_{3}$ de carácter evolutivo. Hallazgo com. patible con necrosis de pared posteroinferior del miocardio (figura 1a).

El caso 9 pesó al nacer $3000 \mathrm{~g}$, Apgar 0 en el primer min y 3 en el quinto. Presión arterial sistólica $50 \mathrm{mmHg}$ y presión venosa central $9 \mathrm{~cm}$ de $\mathrm{H}_{2} \mathrm{O}$. La telerradiografia de tórax mostró un inriltrado intersticial difuso, sin cardiomegalia. CPK total $1430 \mathrm{UL} / 1$ y CPK-MB 53,1 UI/I $(3,7 \%)$, siendo las actividades a las 48 horas de vida 930 y $71,7(7,7 \%)$ respectivamente. EI ECG mostró alto voltaje de los complejos ventriculares derechos en $V_{1}$ y $V_{5}$ y desnivel negativo del segmento $S T$, de $V_{2}$ a $V_{5}$, alteraciones electrocardiográficas compatibles con isquémica subcndocárdica difusa de la pared anterjor del ventrículo izquierdo (figura $1 \mathrm{~b}$ ).

El paciente de] caşo 15 pesó al nacer $3080 \mathrm{~g}$. Apgar 1 y 3 ( 1 y 5 min). Presiones arterial sistólica $60 \mathrm{mmHg}$ y venos central $10 \mathrm{~cm}$ de $\mathrm{H}_{2} \mathrm{O}$. La telerradiografía de tórax no mostraba alteraciones. CPK total $400 \mathrm{UI} / 1$ y MB $49 \mathrm{UI} / 1$ $(12,3 \%)$. En el trazado electrocardiográfico se

Tabla

Actividades séricas (promedios y desviación estándar) de creatina fosfoquina sa e isoenzima MB, deshjdrogenasa láctica, transaminasa glutámico oxaloa cética y pirúvica en recién nacidos con așfixia, daño mjoḉr djco y controles sanos

\begin{tabular}{|c|c|c|c|c|c|c|}
\hline Grupo & $\begin{array}{r}\text { CPK } \\
\text { (UH/ } 1\}\end{array}$ & $\begin{array}{c}\mathrm{CPK} \cdot \mathrm{MB} \\
(\mathrm{UI} / \mathrm{t})\end{array}$ & $\frac{\% \mathrm{CPK}-\mathrm{MB}}{\mathrm{CPK}}$ & $\underset{(\mathrm{UH} / \mathrm{h})}{\mathrm{DHL}}$ & $\begin{array}{l}\text { TGo } \\
\text { (UI } / 1)\end{array}$ & $\begin{array}{c}\text { TGP } \\
\text { (UI/A) }\end{array}$ \\
\hline $\begin{array}{l}\mathrm{RN} \\
\text { controles (16) }\end{array}$ & $427 \pm 284$ & $24 \pm 12$ & $?, 9$ & $544 \pm 148$ & $31 \pm 17$ & $15 \pm$ \\
\hline $\begin{array}{l}\mathrm{RN} \\
\text { asfixtados (13) }\end{array}$ & $598 \pm 493$ & $32 \pm 18$ & 9,2 & $643 \pm 230$ & $42 \pm 20$ & $23 \pm 16$ \\
\hline $\begin{array}{l}\text { RN } \\
\text { asfixiados (3) } \\
\text { datio miocárdico }\end{array}$ & $786 \pm 561$ & $45 \pm 10$ & 7,4 & $567 \pm$ & $43 \pm 17$ & $21 \pm$ \\
\hline
\end{tabular}



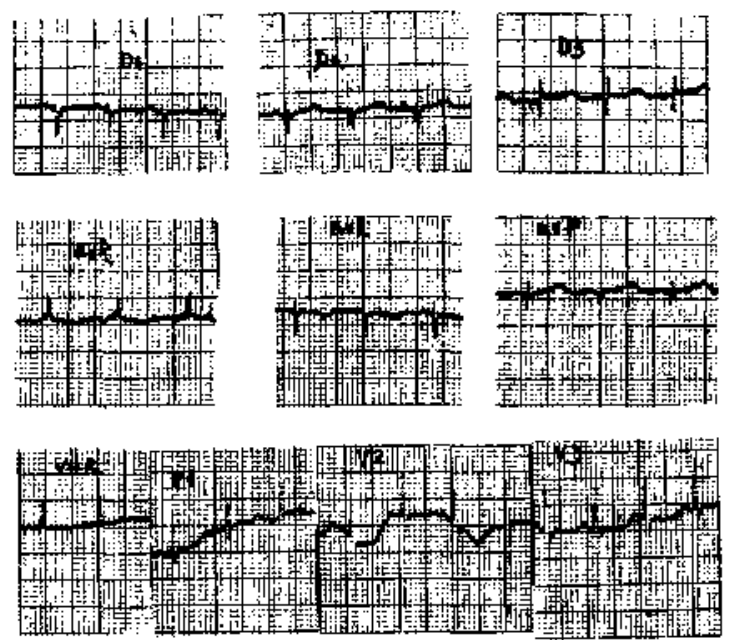
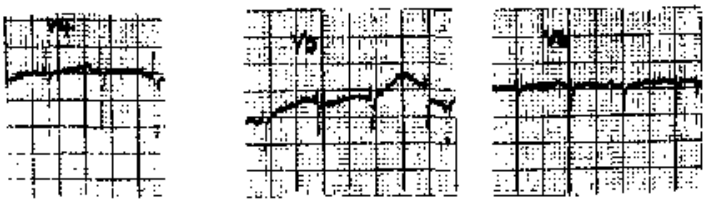

a

Figura 1: a) Caso 3. Ritmo sinusal, frecuencia 120 . min, desviación hacia be derecha del eje de QRS (180\%). Onda $Q$ anormal en $D_{2}, D_{3}$ y avF. Elevación de ST en $D_{3}$ ondas $R$ embrionarias en $V_{5}$ y $V_{6}$. Sugerente de necrosis de pared posterior, b) Caso 9. Taquicardia si-

registraron extrasístoles ventriculares en $\mathrm{V}_{4}$, onda $\mathrm{qR}$ en $\mathrm{D}_{3} \mathrm{y}$ avF, QT largo $\mathrm{y}$ ondas $\mathrm{T}$ aplanadas en $V_{1}$ a $V_{4}$ y negativas en $V_{5}$ y $V_{6}$, trazado compatible con daño miocárdico isquémico (figura 2a). La evolución electrocardiográfica a los 23 dias de vida mostraba ondas QS en $D_{2}-D_{3}$ y avF, indicando isquemia de pared posteroinferior (figura $2 b$ ).

\section{Comentario}

Los recién racjdos asfixiados con signos electrocardiográficos de daño míocárdico isquémico eran de término, con peso adecuado a la edad gestacional, no tenían evidencia de alteración cardíaca en el examen clínico, y sus presiones arterial y venosa eran normales. La frecuencia de isquemia miocárdica transitoria en el recién nacido con asfixia severa es variable. Farrú ${ }^{7}$ la describió en $84 \%$ de sus casos, a través de alteraciones en los ECG de las primeras 24 horas de vida; sin embargo, en sólo el $17 \%$ de ellos
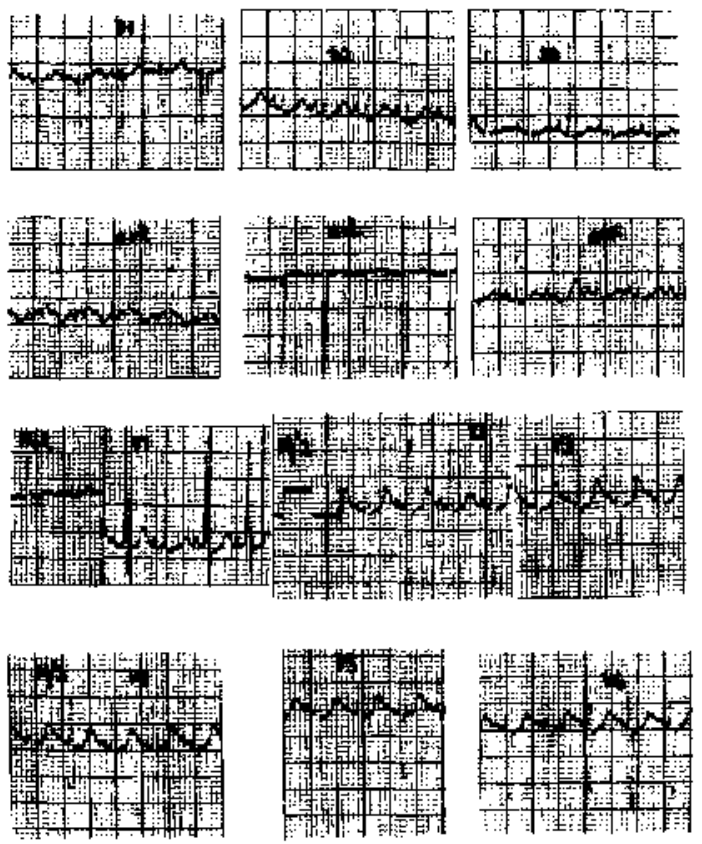

b

nusal, Eje de $Q R S+135^{\circ}$. Alto voltaje de $R$ en $V_{1}$ y $S$ en $V_{5}$. Desnivel negativo de $S T$ de $V_{2}$ a $V_{5}$. Sugiere isquemia subendocárdica difusa de la pared anterior del ventrículo izquierdo.

hubo signos de necrosis de la pared. En cambio Pertman $^{8}$ observó sólo $8 \%$ de anormalidades electrocardiográficas en sus pacientes, en los que la frecuencia de daño miocárdico se elevó a $28 \%$ cuando el diagnóstico se efectuó a través de ultrasonografía modo $\mathrm{M}$ y bidimensional, siendo las alteraciones más frecuentes ventrículo derecho dilatado, regurgitación tricuspídea, cortacircuito atrial y anormalidades ventriculares izquierdas. En nuestra casuística la frecuencia de compromiso miocárdico fue más aproximada a lo observado por Perlman.

Es difícil interpretar los signos de isquemia miocárdica en el ECG en las primeras 24 horas de vida $^{\text {s-11 }}$, debido a que pueden observarse ondas $T$ aplanadas 0 invertidas en las derivaciones precordiales en las horas inmediatas al nacimiento, en recién nacidos normales y con asfixia, sin compromiso miocárdico, a las que se suma onda QR en las derivaciones clásicas, variaciones electrocardiográficas que no muestran ninguna significación clínica. Por este motivo los cambios en la onda $\mathrm{T}$, como demos. 

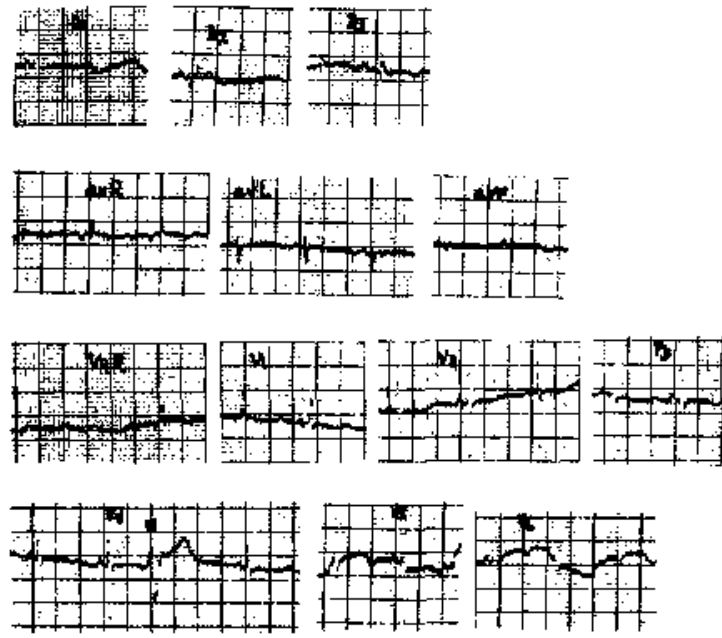

a

Figura 2: Caso 15. a) Trazado inicial. Eje de QRS1800. Extresístoles ventriculares $\left(\mathrm{V}_{4}\right)$. QT largo, $\mathrm{T}$ aplanadas, negativas en $V_{5}$ y $V_{6}$. Sugiere daño mio cárdico isquémico de a pared lateral del ventrículo izquierdo. b) Trazado de control a los 23 día $\mathrm{S}$ ondas $T$ negativas en $\mathbf{D}_{2}, \mathbf{D}_{3}$ y avF. Alteraciones de la repolarización de

tración de isquemia miocárdica, son relevantes sólo después del primer día de vida, siendo valedera la sospecha de daño miocárdico a las pocas horas de nacer cuando hay alteraciones del segmento ST u ondas $Q$ anormales.

En el estudjo enzimático las cifras de CPK total en nuestros casos fueron semejantes a las anteriormente publicadas ${ }^{12,13}$ y las de CPK-MB con controles y recién nacidos con asfixia sin dano miocárdico en el primer día de vida fueron bastante más elevadas que las reportadas por Nelson $^{13}$, pero similares a las de Cuestas ${ }^{14}$ en neonatos normales en las primeras 24 horas de vida. Dado que el número de recién nacidos con daño miocárdico fue muy pequefio, con importante dispersión de los valores entre ellos, no podemos concluir acerca del comportamiento de la CPK-MB en cifras absolutas o como por ciento de la CPK total, aunque su actividad promedio no mostró la elevación descrita por Nelson.

El hallazgo de hemorragjas miocárdicas focales en músculos papilares y septum del paciente fallecido concuerda con lo descrito por otros autores en casuísticas mayores de recién nacidos con asfixia u otro tipo de estrés en las primeras horas de vida, que fallecieron durante la primera
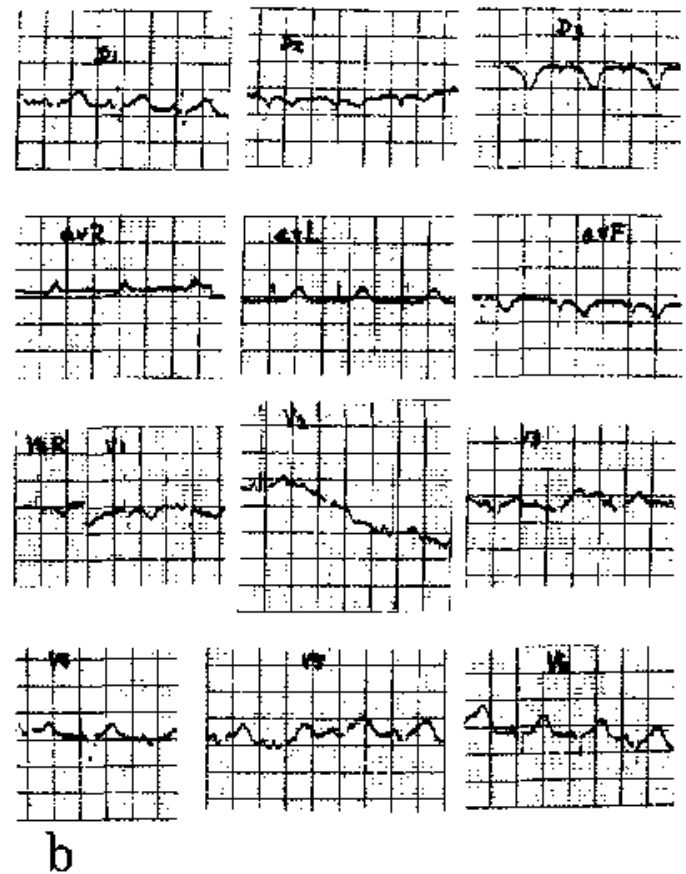

$V_{5}$ y $V_{6}$ han desaparecido. Compatible con daño miocárdico isquémico.

semana postnatal, donde hasta $38 \%$ de los casos tenían uno o más focos de necrosis, siendo ei sitio más comúnmente afectado el subendocar. dio de los músculos papilares anteriores del ventrículo derecho ${ }^{\text {ts }}$. También pueden afectarse los músculos papilares del ventrículo izquierdo y existir lesiones bilaterales ${ }^{16}$. La mayoría de los neonatos con injurja hipóxica severa estudiados por nosotros evolucionó clínicamente con un síndrome de dificultad respiratoria $(88 \%)$. Sólo la evidencia electrocardiográfica de isquenia 0 necrosis permitió determinar en tres de ellos un compromiso miozárdico evolutivo. Esta observación coincide con la descripción previa de dos formas de presentación clínica de ios neonatos que cursan con daño miocárdico: el síndrome de dificultad respiratoria "simple" asociado con alteraciones electrocardiográficas y el síndrome de dificultad respiratoria "complejo" en el cual, a los cambios en el ECG, se suman signos de insuficiencia cardíaca ${ }^{2-3}$. En todos nuestros niños observamos la primera de dichas categorías.

La dificultad respiratoria que se observa en los recién nacjdos asfixiados está determinada por aumento en la presión capilar pulmonar y la permeabilidad endotelial, secundarias 
hipoxja y acidosis ${ }^{17,18}$, que causan edema pulmonar intersticial; éste a su vez altera la mecánica pulmonar, produciendo disminución de la distensibilidad, aumento de la resistencia al flujo aéreo ${ }^{19,20}$, trastorno de la relación ventilaciónperfusión y disminución del volumen corriente $y$, como consecuencia, mayor hipoxemia ${ }^{21}$.

La otra forma de presentación de los recién nacidos con daño mjocádico secundario con dificultad respiratoria asociada a insuficiencia cardíaca no fue observada en nuestros casos. Esos pacientes tienen, además de dificultad respiratoria, grados variables de cianosis, cardiomegalia, ritmo de galope y hepatomegalia. También puede haber insuficiencia mitral o tricuspidea, debida a disfunción de los músculos papilares $^{2-5}$, auscultándose en estos casos soplos sistó licos en los focos correspondientes. Su forma más severa es ef choque cardiogénico, descrito tarnbiên en recién nacidos pretérmino ${ }^{\circ}$, caracterizado por signos de insuficiencia cardíaca, hipotensión arterial, disminución de la perfusión periférica, prolongación del intervalo R-P, marcada elevación de los niveles de ácido láctico y de la presión venosa central, cuya medición es de vital importancia, ya que la innecesaria expansión de volumen agravaría la falla cardíaca. Los neonatos de esta manera afectados suelen responder satisfac. toriamente a la administración de agentes inotrópicos tales como dopamina o dobutami. $\mathrm{na}^{22-24}$.

El daño miocárdico secundario a la asfixia aguda (hipoxia y acidosis) se debería a aumentos de las resistencias vasculares pulmonar y siste. mica, del trabajo ventricular ${ }^{18,25}$ y del cansumo de oxígeno y a disminución del débito coronario, afectando particularmente el subendocardio del ventrículo derecho y la porción posterior del ventrículo izquierdo. Estos, junto con la estimulación simpática que normalmente ocurre con la asfixia $^{25-27}$, producirían aumento significativo de la presión sistólica intramiocárdica a niveles que exceden la presión aórtica, reduciendo aún más el riego coronario, hasta sobrepasar los mecanismos compensatorios normales y desencadenar isquemia significativa, con daño anóxico y necrosis.

Este estudio demuestra la importancia de efectuar electrocardiogramas seriados en los neonatos con asfixia grave, ya que la disfunción cardíaca puede manifestarse como un síndrome de dificultad respiratoria, cursando inadvertida la lesión miocárdica secundaria a la asfixia. El conocimiento de este tipo de daño debe conducir a acciones terapéuticas más eficaces y oportunas que mejorarán la evolución y sobrevida de estos recién nacidos.

\section{Resumen}

Se estudian prospectivamente dieciséjs recién nacidos con asfixia grave, con el propósito de evaluar el compromiso miocárdico y su forma de presentación clínica. En tres RNT AEG $(18,7 \%)$ se diagnosticó daf́o miocárdico a través de electrocardiogramas seriados realizados durante las primeras 72 horas de vida. Dos de ellos evidenciaron signos de isquemia subendocárdica difusa y el tercero un trazado compatible con necrosis de pared posteroinferior del ventrículo izquierdo. La medición de la creatinfosfoquinasa y su fracción MB no fue útil para determinar lesión miocárdica. Los tres neonatos evolucionaron con un sindrome de dificultad respiratoria sin insuficiencia cardíaca. Uno de ellos falleció, encontrándose en el estudio histo. patológico hemorragias focales en los músculos papilares y septum. Este estudio demuestra la importancia de efectuar electrocardiogramas seriados en los neonatos con asfixia grave, puesto que la disfunción cardíaca puede cursar en forma inadvertida, manifestándose como un síndrome de dificultad respiratoria.

(Palabras claves: miocardio, isquemia, asfixia neonatal.)

\section{Referencias}

1. Burnard, E.: James, L.: Failure of the heart after undue asphyxia at bith. Pediatrics 1961; 28: 545-565.

2. Rowe, R.; Hoffman, $T$.: Transient myocardial ischemiz of the newborn infant: $A$ form of sovere cardiorespiratory distress in full-term infant. J Pediatr 1972;81: 243-250.

3. Rowe, R.: Izukawa, T.: Nonstructural heart disease in the newborn. Arch Dis Child 1978; 53: $726 \cdot 730$,

4. Rowe, R.; Finley, J.; Gidoy, D.; Dische, M.; Jimenez, $C$; Change, $G$.: Myocardial ischaemia in the newborn. In Godman M.J., Marquis, R.M. Paediatric Cardiology Vol 2: Heart Disease in the newborn. Churchill Livingstone, Edinburgh: 1979; pp. 8714 .

5. Bucciarelli, R.; Netson, R.; Eganll, E.; Eirzmon, D.; Gessner, I.: Transient tricuspid insufficiency of the newborn: A from of myocardial dysfunction in stressed newborn. Pediatrics 1977;59: 330-337. 
6. Cobal, L.: Devaskar. U.: Siassi, B.; Hodgman, J.; Emmanouilides, G.: Cartiogenic shock associated with perinatal asphyxia in preterm infant. J Pediatr 1980 ; 96 : $705-710$.

7. Farrú, O.; Rizzardini, M.; Guzmán, $N$.: Isquemia transitoria del recién nacido. Rev Chil Pediatr 1987 ; 58: $121-126$.

8. Periam, I.; Tack, E.; Mortin, T,; Shacketford. G.; Amon, $E_{1 .}$ Acute systemic organ injury in term infants after asphyxia. Ann J Dis Child 1989. 143: $617-620$.

9. Kittermon, J,; Phibbs, R.; Tooley. W.: Catheterization of umbilical vessels in newborn infants. Pediatr Clin Notth Am 1970; 17: 895-912.

10. Hait. G.; Gasul, B.: The evolution and significance of $\mathrm{T}$ wave changes in the newborn during the first seven days of life. Ann J Cardiol 1963; 12: 494-503.

11. Jedeikin, R,: Primhak, A.: Shennan, A.: Swyer, P., Rowe, $R$.' Serial electrocardiographic changes in healthy and stressed neonates. Arch Dis Child $1983 ; 58$ : 605-611.

12. Gilboa, $N_{\text {.; }}$ Swanson, $J_{\text {. }}$ Serum creatine phosphokinase in normal newborn. Arch Dis Child 1976 51: 283-285.

13. Nelson, R., Bucciarelli, R, Eitzman, D.; Egan, E.; Gessner, I.: Serum creatine phosphokinase MB fraction in newborn with transient tricuspid insufficiency. N Engl J Med 1978; 298: 146-149.

14. Cuestos, R.: Creatine kinase jsoenzymes in highrisk infants. Pediatt Res 1980; 14: $935-938$.

15. Donnelly, $W$; Bucciarelli, $R$, , Nelson, $R$.: Ischemic papillaty muscle necrosis in stressed newborn jnfants. J Pediatr 1980; 96: 289-294.

16. Setzer, E.; Ermocilla, R.; Tonkin, I.; John, E. Sansa, M.; Cassady, G.: Papiltary muscle necrosis in a neonatal autopsy population: Incidence and associated clinical manifestation. J Pediatt 1980; 96: 289-294.

17. Cohn, H., Sacks, E.; Heymann, M.; Rudolph, A.: Cardiovascular responses to hypoxemia and acidemia in fetal lambs, Am J Obstet Gynecol 1974; $120: 817822$.

18. Rudoiph, $A .:$ The fetal circulation and its response to stress. J Dev Physiol 1984; 6: 11-16.

19. Bancalari, A.; Enriquez, G.; Oyarzún, M.; Quijada, D.: Nî̃os portadores de comunicación interauricular e interventricular: Función pulmonar y flujo de vías aéreas. Rev Med Chil 1981; 109: 614-620.

20. Michel, R.; Zoccht, L. Rost, $A$. et al.: Does interstitial lung edema compress airways and arteries? A morphometric study. J Appl Physiol $1987 ; 62: 108-115$

21. Bland, R.D.: Edema formation in the newborn iung. Clin Perinatol 1982;9: 593-611

22. Driscoll, D.; Gillette, P.; Lewis, R.; Hartley, C.; Schowartz, A.: Comparative hemodynamic effects of isoproterenol, dopamine and dobutamine in the newborn dog. Pediatr Res 1979; 13: 1006 1009 .

23. Driscoll, D.; Gillette. P.; McNamara, D.: The use of dopamine in children. J Pediatr 1978; 92: 309-314.

24. Jewit, D.; Mitchell, A,r Birkhead, J, Dollery, C.: Clinical cardiovascular pharmacology of dobutamine; a selective motropic catecholamine. Lancet 1974; 2 : 363-365.

25. Dawing, $S_{\text {; }}$ Talner, $N$,; Gardner, $T$.: Influences of left ventricular of hypoxemia and acidemia. Am J Physiol 1966; 210: 1327-1331.

26. Behrman, R.; Lees, M, Petersen, E.; Lannoy, C.; Seeds, A.: Distribution of the circulation in the normal and asphyxiated fetal primate. Am J Obstet Gynecol 1970;180:959-961.

27. Jones, $C_{\text {.; }}$ Robinson, $R_{\text {. }}$ : Plasma catecholamines in fetal and adult sheep. J Phisiol 1975; 248: 15-20.

28. Boddy, K.; Jones, C.; Manrell, C.; Ratcliffe, $J$; Robinson, $J$ : Changes in pksma ACTH and corticosteroid of the maternal and fetal sheep during hypoxia. Endocrinology 1974; 94: 588 592 . 